cernod ; assay of soil copper, employing the fungus Aspergillus niger; role of mycorrhizæ in tres nutrition, and studies of fertilizer requirements on soils employing indicator plants grown under glasshouse conditions.

In the past, soil experimental stations have been designed to deal with immediate problems, and many of the more straightforward have been success- fully solved. Problems are now becoming more complex as the soils are utilized more intensively; hence there is a growing need for basic knowledge to be able to assess, solve and, in some cases, anticipate problems. This new experimental station in New Zealand is designed to supply this fundamentel information and so assist the agriculture and forestry of the future.

\title{
A CHALLENGE TO COLORIMETRY
}

$\mathrm{T}$ HE S'mall Physics Theatre at the Imperial College of Science and Technology was filled to capacity on December 12, 1956, when the Physical society Colour Group held a symposium on "Colorimetry : its Errors and Accuracy". An assess ment of the reliability of coleur measurements was manifestly of great interest, and many industries were represented in the audience.

'The problem basically is this: On the trichromatic system of colour measurement established in 1931 by the International Commission on Illumination (C.I.E.), a surface colour can be specified by its chromaticity co-ordinates, $x$ and $y$, and its luminance factor $\beta$. Direct measurement of these quantities by three-colour matching is too inaccurate for most commercial applications, since the matching of lights of markedly different spectral composition brings out individual differences of colour vision. Other methods have therefore been developed, including (a) a six-stimulus visual colorimeter in which an approximate spectral energy match is established and the effect of observer differences therefore reduced; $(b)$ photoelectric colorimeters employing a photo-cell in conjunction with selected colour filters to give spectral sensitivity curves approximating to the three spectral distribution curves of the C.I.E. standard observer; and (c) photoelectric spectrophotometers in which the spectral reflexion curve is measured through the visible spectrum, and the colour specification calculated using the colour mixture data of the standard observer as tabulated by the International Commission on Illumination. Yet none of these methods is at present capable of specifying a colour to a limit of accuracy that cannot be exceeded by the remarkable capacity of the eye to discriminate between two colours of nominally the same specification, when compared side by side in a good light.

In principle, the spectrophotometric method gives the most absolute specification, since the determina. tion of the reflexion factor at each wave-length is unaffected by the spectral sensitivity of the observer or photo-cell. Nevertheless, the accuracy of the result may be limited by the rather small amount of light that is sometimes available for measurement, and if an integrating sphere is used for increasing this amount, then a new uncertainty is introduced because the integrated reflexion may differ from the more directional reflexion employed in visual examination of the specimen. Other possible sources of errer in spectrophotometry include stray light, finite slit-width, limited accuracy of photometric control or linearity of photoelectric response, and uncer. tainty in the specification of the white reference standard.

The magnitude of these inaccuracies was studied by circulating six coloured tiles, supplied by the
British Ceramic Research Association, to a number of research laboratories where reflexion spectrophotometry is practised, and Prof. W. D. Wright (Imperial College) presented a comparison of the results to the meeting. In all, ten different instruments had been used, including five General Electric (Hardy) recording spectrophotometers and five non-recording instruments. Two of the latter employed double monochromators and three single monochromators. The tiles chosen for the test were of various colours and, more important, various surface characteristics, described as glossy, semi-matt and matt, although the polar reflexion curve of the matt tile showed a fairly broad specular component. One of the tiles had a very low reflexion (of the order of 1 per cent) in the green part of the spectrum and it was a tribute to the sensitivity of modern photoelectric devices that in this case the differences in the results from one instrument to another were generally less than 0.1 per cent in reflexion. For the other tiles, the reflexion of which ranged from about 20 to 80 per cent, the spread was of the order of 1 or 2 per cent.

It was noted that for five of the tiles, the average readings with General Electric recording spectrophotometers were $0 \cdot 5-1$ per cent lower than the average of the non-recording instruments, and this difference was attributed to the inclusion in the integrating spheres of the light diffusely rellected at large angles to the normal, whereas the non-recording instruments collected the light within a fairly narrow cone centred around the normal to the surface or, in the case of a Beckman spectrophotometer, in an annular cone at $45^{\circ}$ to the normal. On the other hand, in the case of the so-called matt tile, the General Electric spectrophotometer readings were some 2 per cent higher, apparently due to the inclusion of some of the broad specular component mentioned above, in spite of the insertion in the integrating sphere of the black cap intended to absorb the specular reflexion. Some of the other differences could be traced to errors in the values for the reference white against which the tiles were compared. The ultimate standard is, by definition, a white magnesium oxide surface, but the discussion revealed some divergence of practice in the preparation of such a surface exactly to specification

On the assumption that the average values from the five non-recording instruments were the most nearly correct, the measurements made at the National Physical Laboratory using a Müller-Hilger 'Uvisir' double monochromator were the most accurate, with the results from the Paint Research Station using a Beckman spectrophotometer a close second. While the agreement among the different instruments was regarded by the instrumentalists at the meeting as quite gratifying, the practical colour 
men made it clear that they needed something a good deal better.

The requirements in industry were discussed in a paper by Miss D. L. Tilleard, who described experiments carried out at the Paint Research Station on a set of nearly identical panels prepared by the progressive addition to a standard paint of small amounts of tinting paints of different colours. The panels were measured on the Beckman spectrophotometer and were also compared by experienced observers, under good conditions of daylight illumination, with the panels touching on a long contact line. Under these good viewing conditions, chromaticity differences of 0.0003 or even less could be distinguished in a light biscuit series of panels and differences of less than 0.01 in luminance factor. Commercial tolerances for close matching are larger than this, but may still be as small as 0.001 in chromaticity. This compares with a precision of measurement of the order of 0.0004 in $x$ or $y$ as given by the standard deviation of a number of repeat measurements with the Beckman spectrophotometer, which thus has a differential sensitivity comparable with the closest commercial tolerances.

The same theme was developed further in a paper by Mr. P. S. Williams (Imperial Chemical Industries Paints Division), who compared results on the General Electric recording spectrophotometer and Librascope computer, the Donaldson six-filter colorimeter and the 'Colormaster' differential colorimeter developed by Glasser at the Dupont Company in the United States. When tested on various specimens, the two latter gave results in close agreement to each other, but differing slightly from the former on account of the special illuminating and viewing arrangements, to which reference has already been made. Changes in the spectral transmission of the filters with temperature were found to be one source of error in the Donaldson instrument, while the highest precision with the differential colorimeter (possibly as high as the smallest detectable difference by eye) is only achieved when comparison is made between samples of fairly similar spectral reflexion. This instrument uses a split beam to illuminate a standard and comparison surface, with two photo-cells (plus filters) to form a null balance bridge. Mr. J. W. Perry (Hilger and Watts, Ltd.) discussed the factors which must limit the accuracy of a colorimeter employing filterscreened photo-cells, with particular reference to the Hilger photoelectric tristimulus colorimeter. The fundamental difficulty is, of course, to obtain the correct sensitivity curves, but except for surface colours with irregular spectral reflexion curves, Perry considered that an accuracy of \pm 0.005 could be achieved for a very large range of chromaticities with the Hilger colorimeter. Used as a differential instrument, the precision would be very much higher than this.

As the discussion on all four papers developed, one of the questions asked was, "Where do we go from here ?" The chairman, Mr. R. G. Horner, promised that a future meeting would be held on colour tolerances, to provide an opportunity for the colour industries to define in more detail the limits to which they need to work and to consider the best form in which tolerances should be expressed. One particular point which requires clarification is whether the high discrimination which can be achieved in differential colorimetry needs to be matched by a corresponding precision and accuracy in absolute colour measurements. Evidently if the components of some product are manufactured at different parts of a works or at different times, the manufacturer must control the colour of each component to such an accuracy that they will appear to match when assembled together. Whereas the customer will be able to make a differential and highly critical judgment, the manufacturer may be denied this advantage during the actual production process. In principle, colorimetry is just the tool the manufacturer needs to assist him in this task. In practice, it looks as if the tool still requires some sharpening.

W. D. WRIGHT

\section{NEWS and VIEWS}

Benjamin Franklin Medal of the Royal Society of Arts

THE Council of the Royal Society of Arts has estublished a new award, to be known as the Benjamin Franklin Medal, which is to be made annually "to individuals who have attained early distinction, with promise of future achievement, in the promotion of arts, manufactures and commerce". The association of the Medal with the name of Benjamin Franklin is due to the fact that it was instituted in the year (1956) in which occurred not only the 250th anniversary of Franklin's birth, but also the 200th anniversary of his election to membership of the Society. The medal has been designed by Mr. Christopher Ironside. The first award of the medal is to Prof. F. C. Williams, professor of electrical engineering in the University of Manchester, "for his contributions to electrical engineering".

Prof. F. C. Williams, O.B.E., F.R.S.

Dr. F. C. Willitams, as he then was, joined the staff at Bawdsey Research Station from the Univer- sity of Manchester in March 1939 and was continuously employed throughout the War on the development of radar equipment. During this period, he was himself primarily responsible for the fundamental concepts on which many vital radar systems and devices and automatic servo-mechanisms were based. Dr. Williams resigned from Government service at the end of 1946 and since that time has held the chair of electrical engineering in the University of Manchester. During this post-war period his name has become associated with pioneering work on automatic electronic digital computers. In this field, among his more noteworthy contributions is an electrostatic information store, based on cathoderay tube techniques, of extreme elegance and technical interest. Indeed, the first commercial highspeed electronic digital computer to become available in the United Kingdom was fundamentally based on Prof. Williams's cathode-ray tube information store. More recently, he has turned his attention to the design of rotating electrical machinery and is at present working on an original design for an induction motor, the speed of which is capable of being continuously varied over a wide range. 